oxygen may also be used as adjuvant treatment in refractory cases, though its effectiveness is controversial. ${ }^{4}$ As far as we are aware necrotising fasciitis has not previously been described following a paronychia. What is more unusual is the anatomical basis for the distribution of the rash, which we are currently unable to explain.

In summary, we have presented an unusual manifestation of a common condition and reaffirm the need for early drainage of all paronychias presenting to the $A \& E$ department.
We would like to thank Emma Taylor and Andrew Rolland, of the Medical Photography Department, St George's Hospital Medical School, for their kind assistance.

1 Moran GJ, Talan DA. Hand infections. Emerg Med Clin North Am 1993;11:601-19.

2 Saccone PG, Rayan GM. Subungual malignant degeneration following chronic perionychial infection. Orthop Rev 1993;22:623-6.

3 Blankstein A, Nerubay J, Lin E, Keren G, Freidman B, Horowski H. Septic arthritis of the sternoclavicular joint. Orthop Rev 1986;15:440-2.

4 Shupak A, Shoshani O, Goldenberg I, Barsilai A, Moskuna $R$, Bursztein S. Necrotizing fasciitis: an indication for hyperbaric oxygenation therapy? Surgery 1995;1 18:873-8.

\title{
Infratemporal and temporal fossa abscess complicating dental extraction
}

\author{
M S Diacono, A R Wass
}

\begin{abstract}
Abscess formation in the infratemporal and temporal fossae is rare. Their presentation to accident and emergency departments is unusual and consequently may cause problems with diagnosis. Once diagnosed, treatment should be aggressive with intravenous antibiotics and surgical drainage.

(F Accid Emerg Med 1998;15:59-67)
\end{abstract}

Keywords: dental extraction; temporal fossa; infratemporal fossa; abscess

\section{Case report}

A 57 year old married white male presented to the accident and emergency (A\&E) department complaining of severe shooting pains on the left side of his face, and difficulty in eating, opening his mouth, and sleeping. Three weeks previously his general dental practitioner extracted his upper left first molar tooth under

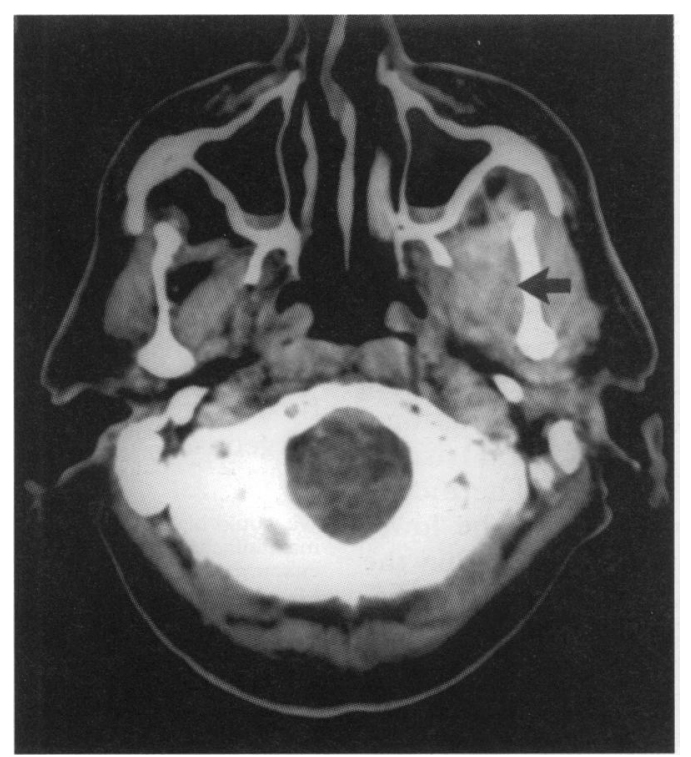

Figure 1 Axial computerised tomography showing lesion in left infratemporal fossa. local anaesthetic. His symptoms developed slowly after this procedure, despite two courses of antibiotics from the practitioner.

Examination within the $A \& E$ department showed him to be pyrexial $\left(40^{\circ} \mathrm{C}\right)$ with signs of systemic sepsis. He had severe trismus, and light palpation of the left side of his face triggered sharp radiating pains in all dermatomes of the left trigeminal nerve. The left temporal area was warm, red, and mildly swollen. All branches of his facial nerve functioned normally. The upper left first molar socket was healing normally. There was no cervical lymphadenopathy.

Occipitomental, lateral facial, and panoral radiography was normal. A full blood count was unremarkable; however, the erythrocyte sedimentation rate was raised at $62 \mathrm{~mm} / \mathrm{h}$. Routine biochemistry studies showed a mild hypokalaemia (plasma potassium $3.2 \mathrm{mmol} /$ litre).

The patient was admitted under the care of the maxillofacial surgeons and prescribed intravenous cofluampicil and metronidazole as well as analgesics.

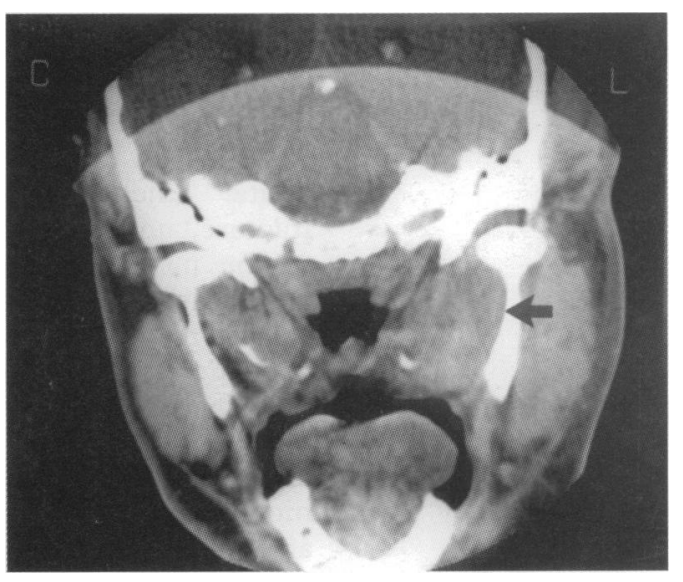

Figure 2 Coronal computerised tomography showing loss of normal anatomical definition in left infratemporal fossa due to lesion. 
Computerised tomography in axial and coronal planes (figs 1 and 2) revealed a mass in the infratemporal and temporal fossae, initially reported as a soft tissue neoplasm. However, over the next 72 hours the temporal swelling increased, became fluctuant, and spread to the preauricular area. Pneumonia and possible mediastinitis developed, which delayed surgical drainage. Blood cultures grew Streptococcus milleri, which was treated with meropenem (500 $\mathrm{mg}$ three times daily). Six days after admission, incision, drainage, and biopsy by an intraoral and temporal route were performed. The patient's toxic state improved dramatically and his neuralgic pains subsided over a few weeks.

The pathology report confirmed the presence of granulation tissue with infiltration of acute and chronic inflammatory cells and no signs of neoplasia. At one year follow up the patient remains pain-free but his mouth opening is limited to $50 \%$ of normal.

\section{Discussion}

The infratemporal fossa is well described. It is an irregularly shaped space below the greater wing of the sphenoid bone (containing the foramen ovale), lateral to the ramus of the mandible and the gap between the zygomatic arch and temporal bone (forming the communication to the temporal fossa). The lateral pterygoid plate forms the medial margin while the maxilla forms the medial aspect of the space. The infratemporal fossa contains the temporalis muscle, medial and lateral pterygoid muscles, the pterygoid plexus of veins, the maxillary artery and its branches, the mandibular nerve and its branches, and the chorda tympani. ${ }^{1}$ The parapharyngeal space lies medially and communicates with the mediastinum.

The temporal fossa is bound superiorly and distally by the temporal ridge, anteriorly by the frontal bone, zygomatic bone, and greater wing of sphenoid, and laterally by the zygomatic arch, and it communicates with the infratemporal fossa inferiorly. It contains branches of the deep temporal artery and is filled by the temporalis muscle. ${ }^{2}$

Infection of the infratemporal and temporal fossae has been attributed to several causes. Odontologically related infections have been most commonly reported. ${ }^{3-5}$ Both maxillary and mandibular third molar infection have been implicated. ${ }^{4}$ Other sources of infection described in these masticatory spaces include dental injections, ${ }^{6}$ maxillary sinus wall fracture, ${ }^{7}$ and haematogenous spread. ${ }^{3}$

The most commonly reported symptoms and signs are pain, fever, malaise, and trismus (which may be partial or total). ${ }^{3}$ The medial and lateral pterygoid muscles and the lower aspect of the temporalis muscle lie within the infratemporal fossa. Infection causes myofasciitis and loss of function, leading to limited mouth opening. Swallowing may be difficult and patients often describe a feeling of fullness in the affected area. External swelling, proptosis, and oedema are normally late presentations. ${ }^{5}$ Trigeminal neuralgia type pains have been reported ${ }^{3}$ : the mandibular branch of the trigeminal nerve exits from the cranial floor through the foramen ovale before traversing the infratemporal fossa; the inflammatory process in this area, as well as in the affected alveolus, may trigger symptoms similar to trigeminal neuralgia. In this case light touch did precipitate sharp stabbing pain; however, trigeminal neuralgia rarely affects patients at night and usually no other signs are present. ${ }^{8}$

The diagnosis of masticatory space infection relies upon a high index of suspicion. Plain radiographs including panoral views may identify the source of infection and soft tissue swelling (in this case radiography was unremarkable except for the extraction site). A neutrophil leucocytosis is usual. Positive blood cultures are a useful diagnostic aid, especially if the organism identified is commonly associated with dental sepsis such as Str milleri. ${ }^{9}$

Computerised tomography or magnetic resonance imaging are usually diagnostic, but it is not always easy to distinguish between abscesses and tumours. If an abscess is suspected, these imaging techniques may guide the surgeon to the best possible site for incision and drainage by identifying the fascial plane in which the abscess lies. ${ }^{3}$

Three basic patterns of infratemporal fossa infection have been identified: first, localisation to the fossa itself; second, superior spread into the temporal fossa; and finally, inferior spread into the floor of mouth, neck, and parapharyngeal spaces despite the presence of distinct fascial barriers. ${ }^{3}$ In the case reported here, infection arising from the maxillary left first molar tooth spread into the infratemporal fossa. As it remained unchecked it spread both superiorly into the temporal fossa and inferiorly into the parapharyngeal spaces towards the mediastinum. The rapid spread of infection (including chest infection) indicates the urgency required for correct diagnosis and treatment of infratemporal fossa infections.

\section{CONCLUSION}

Infratemporal and temporal fossa infection is not commonly seen by A\&E clinicians and might be easily misdiagnosed. A careful medical and dental history is required, as well as oral, facial, and systemic examination. With this information, together with radiographic findings and knowledge of the anatomical structures involved, infratemporal fossa infection can be suspected and management begun by urgent referral to the oral and maxillofacial surgeons.

We would like to thank Mr D Hutchinson, consultant oral and maxillofacial surgeon at Pinderfields Hospital, Wakefield, for encouraging the preparation of this case report on his patient.

1 McMinn RMH, Hutchings RT, Logan BM. Colour atlas of head and neck anatomy. London: Wolfe Medical Publications, 1985:117.

2 Gray H. Gray's anatomy, the classic collector's edition. New York: Crown Publishers, 1977:110-11

3 Hardin CW, Harnsberger HR, Osborn A, Doxey GP, Davis RK, Nyberg DA. Infection and tumour of the masticator space: CT evaluation. Radiology 1985;157:413-7. 
4 Schwimmer A, Roth SE, Morrison SN. The use of computerised tomography in the diagnosis and management of temporal and infratemporal space abscesses. Oral Surg Oral Med Oral Pathol 1988;66:17-20.

5 Banerjee SC. Temporal osteitis and infratemporal space infection following dental extraction. Oral Surg Oral Med Oral Pathol 1966;21:15-18.

6 El-Sheikh MM, El-Hak RY. Infection of the infratemporal El-Sheikh MM, El-Hak RY. Infection of
space. Br J Oral Surg 1972;10:189-92.
7 Weiss BR. Infratemporal fossa abscess; unusual complication of maxillary sinus fracture. Laryngoscope 1977:98: 1130-3.

8 Tyldesley WR. Oral medicine. Oxford: Oxford Medical Publications, 1981:151-2.

9 Stock CJR, Gulabivala K, Walker RT, Goodman JR. Colour atlas and text of endodontics, 2nd ed. London: Mosby-Wolfe, 1995:21.

\section{Pre-Hospital Immediate Care}

\section{"Towards Evidence-Based Pre-Hospital Care" \\ One day conference}

\section{Thursday 5th March 1998}

Commonwealth Institute

High Street Kensington, London W8

Following the success of the first Pre-hospital Immediate Care conference held in March 1997, this second conference will focus on evidence-based pre-hospital care - a method ensuring that clinical decision making is based on the best available evidence.

The conference is aimed at:

- Pre-hospital care and the emergency services

- Emergency doctors and nurses

- Paramedics

- EMTs

- Anyone with a strong interest in pre-hospital care.

\section{Scientific programme}

The scientific programme will be made up of plenary sessions combined with a series of practical workshops. Keynote speakers will address areas of growing importance.

\section{Posters and free papers-call for abstracts}

For authors' instructions and further information please contact Kathryn Sims, BMJ Publishing Group, BMA House, Tavistock Square, London, WC1H 9JP, Tel 0171383 6795, Fax 0171383 6668, Email 101317.477@, compuserve.com

\section{Exhibition}

A commercial exhibition will take place during the conference. Companies or organisations wishing for further information should contact Jane Lewis at the address below.

\section{Registration}

For a registration form and further information please contact Jane Lewis, BMA Conference Unit, BMA House, Tavistock Square, London, WC1H 9JP. Tel 0171383 6605, Fax 0171383 6663, Email JaneLewis@BMA.org.com 